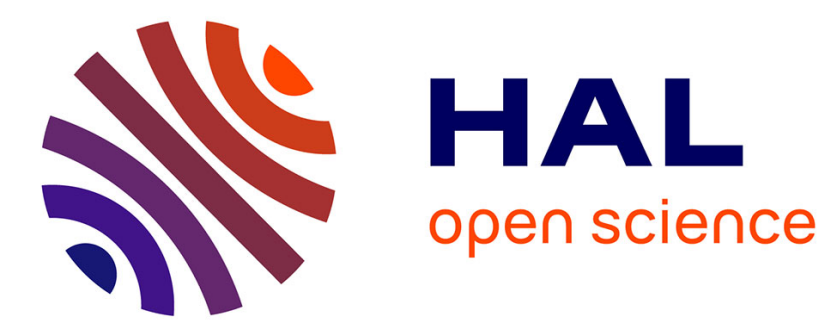

\title{
Influence d'une supplémentation de la ration en lipides sur la qualité du lait chez la vache
}

\author{
M. Doreau, Y. Chilliard
}

\section{To cite this version:}

M. Doreau, Y. Chilliard. Influence d'une supplémentation de la ration en lipides sur la qualité du lait chez la vache. Productions Animales, 1992, 5 (2), pp.103-111. hal-00895966

\section{HAL Id: hal-00895966 https://hal.science/hal-00895966}

Submitted on 1 Jan 1992

HAL is a multi-disciplinary open access archive for the deposit and dissemination of scientific research documents, whether they are published or not. The documents may come from teaching and research institutions in France or abroad, or from public or private research centers.
L'archive ouverte pluridisciplinaire HAL, est destinée au dépôt et à la diffusion de documents scientifiques de niveau recherche, publiés ou non, émanant des établissements d'enseignement et de recherche français ou étrangers, des laboratoires publics ou privés. 
INRA Prod. Anim., $1992,5(2), 103-111$

\section{DOREAU, Y. CHILLIARD}

\section{INRA Laboratoire Sous-Nutrition} des Ruminants

Theix - 63122 Saint-Genès-Champanelle

\section{Influence d'une supplémentation de la ration en lipides sur la qualité du lait chez la vache}

L'incorporation des lipides permet d'augmenter, à moindre coût, la concentration énergétique des rations pour vaches laitières. La production laitière est en général augmentée mais le taux protéique diminué et la composition des matières grasses du lait modifiée, de façon variable suivant la nature du supplément lipidique et le stade de lactation des animaux.

La supplémentation en lipides est souvent pratiquée dans les rations pour vaches laitières. Plusieurs raisons expliquent cette tendance récente. Le coût des matières grasses a diminué et leur permet de concurrencer d'autres sources d'énergie. De plus, leur emploi entraîne l'augmentation de la concentration énergétique des rations. En début de lactation, il serait ainsi possible de limiter le déficit énergétique des vaches fortes productrices (Doreau et al 1987). De plus, on connaît désormais les moyens de limiter les perturbations qu'engendrent les matières grasses sur la digestion des constituants pariétaux des rations : apporter un supplément de calcium

\section{Résumé}

L'enrichissement en matières grasses des rations est couramment pratiqué pour les vaches laitières fortes productrices. L'une des conséquences les plus fréquentes est la diminution de la teneur en protéines, et plus particulièrement en caséines du lait. Les causes en sont encore mal connues. Une partie de cet effet est liée à la dilution des protéines dans un volume accru de lait, car la sécrétion totale de protéines n'est que peu affectée. La diminution du taux protéique est beaucoup plus modérée au début de la lactation, où l'effet positif sur la production laitière est moins net, qu'après le pic de production.

L'incorporation de matières grasses dans les rations a un effet très variable sur le taux butyreux, qui dépend du bilan entre l'augmentation des acides gras longs d'origine alimentaire et la réduction de la synthèse mammaire des acides gras à chaîne courte ou moyenne. La teneur en acides gras polyinsaturés dans le lait n'est presque jamais fortement modifiée, en raison de l'hydrogénation des lipides alimentaires dans le rumen. Seule la technique d'encapsulation des lipides dans une coque de protéines tannées protège les lipides alimentaires de l'hydrogénation ruminale.

Certains types de suppléments lipidiques entraînent une détérioration de la valeur organoleptique des produits laitiers, en particulier par oxydation et rancissement. dans la ration, distribuer des sources de matières grasses perturbant peu la digestion, comme les savons de calcium (Doreau et al 1989).

Chez le ruminant laitier, l'incorporation de lipides dans la ration se traduit par une action variable sur le taux butyreux, par une modification de la composition en acides gras du lait dans la plupart des cas, et par une action généralement négative sur le taux protéique. L'influence sur les matières grasses du lait a été largement étudiée et a fait l'objet de nombreuses revues bibliographiques de synthèse. L'effet négatif sur le taux protéique a été amplement démontré, mais peu analysé. Par ailleurs, très peu de données expérimentales sont disponibles sur l'action des lipides alimentaires sur les fractions protéiques du lait et sa valeur technologique.

\section{1 / Lipides de la ration et matières azotées du lait}

\section{1 / Taux protéique}

Le plus souvent, la supplémentation d'une ration en lipides entraîne une réduction de la teneur en matières azotées du lait. MorandFehr et al (1986), récapitulant 42 expérimentations, ont trouvé un effet négatif dans 30 cas, nul dans 5 cas et positif dans 7 cas.

Le taux protéique diminue même lorsqu'une faible quantité de lipides est ajoutée (Bines et al 1978, Chilliard et al 1992). Jusqu'à présent, la chute de taux protéique lors d'une supplémentation lipidique n'a pu 
être reliée ni au taux protéique du régime témoin, ni à la nature de la ration de base. L'effet de la nature des lipides semble en revanche variable. D'après l'analyse de Sechier et Vérité (non publié) portant sur 54 expérimentations, les plus fortes diminutions de taux protéique seraient observées avec l'huile de soja, les plus faibles avec l'huile de coton et la graine de tournesol, et des diminutions d'amplitude intermédiaire seraient relevées avec le suif, la graine de coton et la graine de soja. Plus généralement, les huiles entraîneraient une plus faible chute du taux protéique que les graines correspondantes, à même niveau de lipides ajoutés (Sauvant et al non publié, résultats de 11 comparaisons directes). Avec différents types de lipides "protégés", le taux protéique diminue en moyenne de $1,3 \mathrm{~g} / \mathrm{kg}$ pour un taux d'incorporation moyen de $740 \mathrm{~g} / \mathrm{j}$ (Chilliard et al 1992, analyse de 65 essais)
A l'examen du tableau 1 et de la figure 1, il apparaît que l'effet négatif sur le taux protéique est plus limité dans les premières semaines de la lactation (avant le pic de lactation, ou phase descendante du taux protéique) que dans les semaines suivantes (après le pic de lactation, ou phase ascendante du taux protéique). Ceci est confirmé par des données de Casper et al (1990) et Hoffman et al (1991) alors que Finn et al (1985) ne l'avaient pas observé.

La quantité de protéines sécrétées est beaucoup moins affectée que le taux protéique par la supplémentation lipidique. Ainsi, Chilliard et al (1992), dans une synthèse d'essais sur des vaches produisant en moyenne $25 \mathrm{~kg} / \mathrm{j}$ (de 10 à $45 \mathrm{~kg} / \mathrm{j}$ selon les essais) montrent qu'avec des huiles végétales protégées (16 essais), qui ne permettaient pas d'accroître la production laitière $(-0,04 \mathrm{~kg} / \mathrm{j}) \mathrm{le}$ taux protéique diminuait de $0,8 \mathrm{~g} / \mathrm{kg}$ et la pro-

\section{L'addition de lipides à la ration conduit à un accroissement de la production laitière, essentiellement après le pic de lactation.}

Tableau 1. Effet du stade de lactation sur le taux protéique et la sécrétion de protéines dans le lait. Ces données récapitulent les résultats obtenus d'une part avant le pic de lactation (ou aux alentours du pic), d'autre part après le pic de lactation, en phase descendante. Les résultats correspondant aux régimes supplémentés en lipides sont les différences par rapport aux résultats des régimes non supplémentés.

\begin{tabular}{|c|c|c|c|c|c|c|c|}
\hline \multirow{2}{*}{ 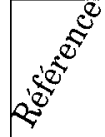 } & \multirow{2}{*}{$\begin{array}{l}\text { Nature du } \\
\text { supplément } \\
\text { lipidique }\end{array}$} & \multicolumn{3}{|c|}{ avant le pic } & \multicolumn{3}{|c|}{ après le pic } \\
\hline & & PL & $\mathrm{TP}$ & $\mathrm{PP}$ & PL & $\mathrm{TP}$ & PP \\
\hline 1 & $\begin{array}{l}\quad- \\
6 \% \text { suif } \mathrm{E} \\
8,5 \% \text { suif } \mathrm{E}\end{array}$ & $\begin{array}{r}35,6 \\
+0,3 \\
-2,0\end{array}$ & $\begin{array}{l}29,4 \\
-2,2 \\
-3,1\end{array}$ & $\begin{array}{r}1040 \\
-60 \\
-200\end{array}$ & $\begin{array}{r}32,3 \\
-0,8 \\
+3,6\end{array}$ & $\begin{array}{l}30,5 \\
-1,7 \\
-0,5\end{array}$ & $\begin{array}{r}960 \\
-100 \\
+140\end{array}$ \\
\hline 2 & $\begin{array}{l}3,5 \% \text { suif } \mathrm{E} \\
7 \% \text { suif } \mathrm{E} \\
10,5 \% \text { suif } \mathrm{E}\end{array}$ & $\begin{array}{r}21,4 \\
+3,9 \\
+2,3 \\
+1,2\end{array}$ & $\begin{array}{l}32,0 \\
-1,1 \\
-1,0 \\
-1,4\end{array}$ & $\begin{array}{r}680 \\
+100 \\
+50 \\
+10\end{array}$ & $\begin{array}{r}21,7 \\
+2,1 \\
+1,9 \\
-4,3\end{array}$ & $\begin{array}{l}32,5 \\
-2,1 \\
-3,6 \\
-3,2\end{array}$ & $\begin{array}{r}700 \\
+20 \\
-20 \\
-190\end{array}$ \\
\hline 3 & $\begin{array}{l}- \\
6 \% \text { graisse d'os } \\
6 \% \text { GC saturées }\end{array}$ & $\begin{array}{r}28,2 \\
+0,8 \\
+0,8\end{array}$ & $\begin{array}{r}31,0 \\
+0,8 \\
-0,5\end{array}$ & $\begin{array}{r}862 \\
+41 \\
+9\end{array}$ & $\begin{array}{r}26,5 \\
+2,6 \\
+1,7\end{array}$ & $\begin{array}{l}28,6 \\
-0,3 \\
-0,8\end{array}$ & $\begin{array}{r}756 \\
+61 \\
+26\end{array}$ \\
\hline $4 a$ & $3,5 \% \mathrm{SC}$ saturés & $\begin{array}{c}30,5 \\
0\end{array}$ & $\begin{array}{r}31,5 \\
+0,6\end{array}$ & $\begin{array}{r}941 \\
+3\end{array}$ & $\begin{array}{r}31,3 \\
+0,5\end{array}$ & $\begin{array}{l}28,8 \\
-1,6\end{array}$ & $\begin{array}{l}909 \\
-46\end{array}$ \\
\hline $4 b$ & $3 \%$ SC saturés & $\begin{array}{r}28,8 \\
+1,0\end{array}$ & $\begin{array}{r}31,4 \\
-0,7\end{array}$ & $\begin{array}{r}903 \\
+5\end{array}$ & $\begin{array}{r}29,5 \\
+1,6\end{array}$ & $\begin{array}{l}29,4 \\
-1,9\end{array}$ & $\begin{array}{l}871 \\
-23\end{array}$ \\
\hline 5 & $\begin{array}{l}3 \% \text { SC saturés } \\
6 \% \text { SC saturés }\end{array}$ & $\begin{array}{l}30,8 \\
-1,1 \\
-3,6\end{array}$ & $\begin{array}{r}28,4 \\
+0,1 \\
-1,4\end{array}$ & $\begin{array}{r}875 \\
-37 \\
-141\end{array}$ & $\begin{array}{r}26,5 \\
+1,5 \\
+1,5\end{array}$ & $\begin{array}{l}29,6 \\
-1,7 \\
-3,3\end{array}$ & $\begin{array}{r}784 \\
-3 \\
-48\end{array}$ \\
\hline 6 & $\begin{array}{l}3,75 \% \text { SC saturés } \\
3,75 \% \text { GE de colza }\end{array}$ & $\begin{array}{r}28,4 \\
-1,5 \\
+0,1\end{array}$ & $\begin{array}{r}28,3 \\
-0,5 \\
+0,3\end{array}$ & $\begin{array}{r}791 \\
-44 \\
+11\end{array}$ & $\begin{array}{r}27,2 \\
+0,1 \\
+1,1\end{array}$ & $\begin{array}{l}29,0 \\
-0,5 \\
-0,7\end{array}$ & $\begin{array}{r}778 \\
-1 \\
+21\end{array}$ \\
\hline
\end{tabular}

PL : production laitière $(\mathrm{kg} / \mathrm{j}) ; \mathrm{TP}:$ taux protéique $(\mathrm{g} / \mathrm{kg}) ; \mathrm{PP}:$ production de protéines $(\mathrm{g} / \mathrm{j}) ; \mathrm{E}:$ encapsulé avec des protéines traitées au formaldéhyde ; GC : graisses cristallisées ; SC : savons de calcium ; GE : graines extrudées.

1 - Macleod et al $1977 ;$ ration $2 / 3$ concentré et $1 / 3$ foin

2 - Bines et al 1978 ; ration à $75 \%$ d'aliment concentré

3 - Chilliard et Doreau, non publié ; ration d'ensilage de maïs

4 - Elmeddah et al non publié ; ration d'ensilage d'herbe ;

$\mathrm{a}$ : concentré riche en amidon, $\mathrm{b}:$ concentré riche en parois

5 - Chilliard et al non publié ; ration d'ensilage de mais

6 - Doreau et al non publié ; ration d'ensilage de maïs. 
duction de protéines de $8 \mathrm{~g} / \mathrm{j}$. Avec des matières grasses saturées, encapsulées ou saponifiées (49 essais), permettant d'accroître la production laitière de $0,95 \mathrm{~kg} / \mathrm{j}$, le taux protéique diminuait de $1,5 \mathrm{~g} / \mathrm{kg}$, mais la production de protéines de $10 \mathrm{~g} / \mathrm{j}$ seulement. Cet effet de "dilution" des protéines dans le lait, qui avait été signalé par Hermansen et Pedersen (1987) à partir d'expérimentations danoises, se vérifie sur des moyennes d'essais, mais une variabilité d'une expérimentation à l'autre subsiste. Il pourrait par ailleurs expliquer en partie le fait que le taux protéique est peu affecté avant le pic de lactation : l'effet des lipides sur la production laitière est alors généralement beaucoup moins net qu'après le pic (figure 1).

Dans la mesure où l'effet de dilution n'explique pas la totalité des effets de la supplémentation lipidique, il est nécessaire de rechercher les causes de cette chute du taux protéique. Deux hypothèses mettant en jeu des mécanismes hormonaux ont été émises:

- Une résistance du tissu mammaire à l'insuline a été suggérée par Palmquist et Moser (1981) : pour des vaches ayant subi une injection de glucose, la vitesse de diminution de la glycémie est plus faible et l'insulinémie plus élevée avec des régimes riches en matières grasses. Cette constatation est toutefois délicate à relier directement au taux protéique du lait.

- A partir de mesures de différences artérioveineuses, Casper et Schingoethe (1989) et Casper et al (1990) ont observé, contrairement à Depeters et al (1989), que l'addition de matières grasses à la ration entraîne une diminution du prélèvement mammaire des différents acides aminés. Cela les a conduits à envisager que l'addition de matières grasses réduirait la sécrétion de somatotropine des vaches.

Ces hypothèses n'ont pas reçu de confirmation jusqu'à présent. L'effet de l'addition de lipides sur les concentrations sanguines d'insuline ou de somatotropine est très variable. Par ailleurs, la chute du taux protéique n'a pas été évitée dans un essai de Lough et al (1988) dans lequel les vaches recevaient de la somatotropine simultanément aux matières grasses. Enfin, des données récentes indiquent qu'une infusion duodénale d'huile ne modifie pas les concentrations de ces hormones en début de lactation, mais accroît la somatotropinémie et réduit l'insulinémie en milieu de lactation, sans modifier la réponse de la glycémie à une injection d'insuline, tout en diminuant fortement le taux protéique (Gagliostro et Chilliard 1991 et Gagliostro et al 1991).

Une autre hypothèse a été plusieurs fois émise : l'apport d'acides aminés à la mamelle serait réduit par la supplémentation lipidique en raison de la diminution de la synthèse de protéines microbiennes dans le rumen. Ceci n'explique qu'une partie des cas observés. En effet, les chutes de taux protéique lors
Figure 1. Effet de l'incorporation de matières grasses dans la ration sur le TP et la production laitière ( $P L)$, avant et après le pic de lactation (résultats INRA de Theix et Orcival).

Les variations représentées sont les différences entre le TP ou la production laitière du lot enrichi en lipides et du lot témoin. Suppléments de lipides : essais 1 et $2: 3-3,5 \%$ de savons de calcium; essai $3: 6 \%$ de matières grasses saturées cristallisées ; essai $4: 6 \%$ de graisse d'os; essai $5: 3 \%$ de savons de calcium; essai $6: 6 \%$ de savons de calcium; essai $7: 3,75 \%$ de savons de calcium ; essai $8: 3,75 \%$ de graines de colza extrudées. Rations à base d'ensilage d'herbe (essais 1,2) ou de mais (essais 3, 4, 5, 6, 7, 8).

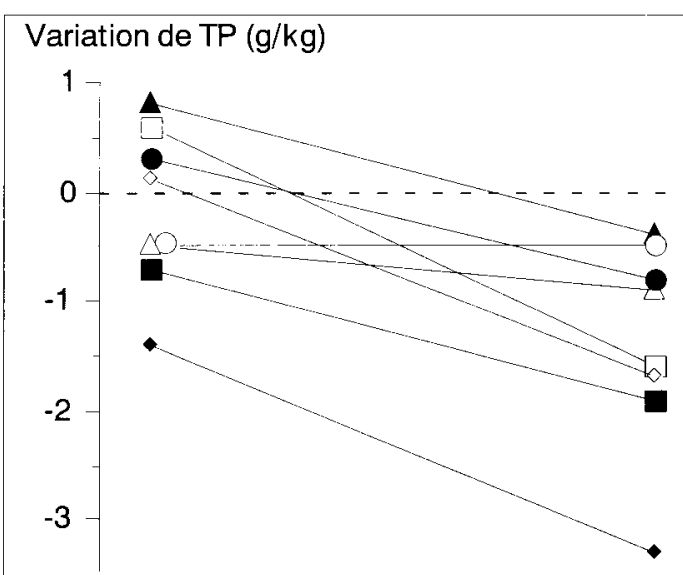

Variation de $P L(k g / j)$

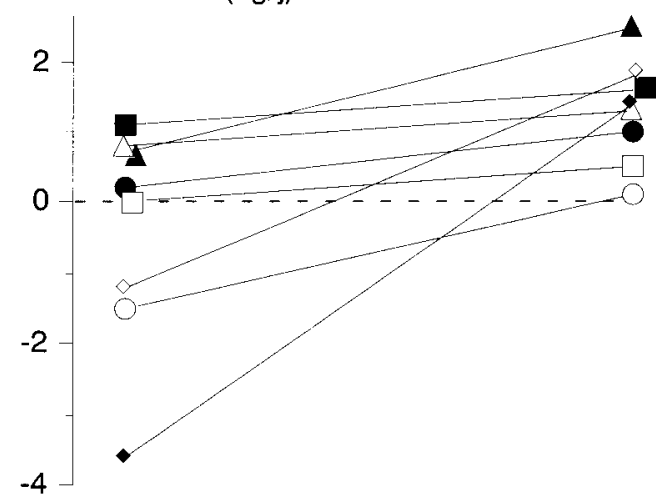

Avant le pic

Après le pic

essai :
$1 \square 2$
$2 \triangle 3 \wedge$
40
$5 \cdot 6$
7
8

d'apport de lipides se produisent même lorsque les vaches sont en bilan azoté largement positif. Par ailleurs, les expériences de digestion montrent que l'addition de lipides ne modifie généralement pas le flux d'azote sortant du rumen (Tamminga et Doreau 1991). On peut toutefois penser que la synthèse de protéines microbiennes est limitée lorsque les lipides sont apportées en substitution aux glucides : il pourrait dans ce cas y avoir une limitation de l'énergie fermentescible nécessaire à la synthèse de protéines.

On a tenté d'éviter les chutes de taux protéique en ajoutant à la ration des additifs permettant parfois d'accroître le taux protéique.
L'effet négatif sur le taux protéique est particulièrement net après le pic de lactation. 
Avec des acides aminés protégés (Canale et al 1990, Chow et al 1990) ou infusés au niveau duodénal (Chilliard et Doreau 1991), il y a eu un effet positif des acides aminés sur le taux protéique mais pas d'interaction ou même une interaction négative entre ceux-ci et les lipides. Au mieux l'effet positif des acides aminés était aussi élevé avec le supplément lipidique qu'avec la ration non supplémentée. Il n'y a pas eu d'interaction positive entre la niacine et les lipides dans un essai de Horner et al (1986), alors que la niacine a permis une augmentation du taux protéique plus importante avec un régime riche en matières grasses qu'avec un régime témoin dans un essai de Driver et al (1990). Ces différentes données nécessitent donc confirmation.

Peu de variations de taux protéique ont été mises en évidence lors d'une supplémentation lipidique chez la chèvre ou la brebis (Sauvant et al 1983, Caja et Guillou 1990).

\section{2 / Fractions azotées}

Il est couramment affirmé que la diminution de la teneur en protéines du lait lors d'une supplémentation lipidique est due à une diminution de la synthèse des caséines. Cette assertion provient des deux premières expérimentations sur les fractions azotées du lait dans le cas de rations enrichies en lipides (Storry et al 1974, Dunkley et al 1977). De nouveaux travaux ont été réalisés depuis 5 ans, essentiellement aux Etats-Unis, et ont été récapitulés sur le tableau 2 . Il apparaît en effet que les diminutions du taux protéique sont explicables par des diminutions du taux de caséines. Mais, comme dans certains de ces essais la production laitière augmentait avec l'incorporation de lipides, la production de protéines varie entre +73 et $-141 \mathrm{~g} / \mathrm{j}$ selon les essais. La production de caséines explique l'essentiel de ces variations, même lorsque l'apport de lipides entraîne un accroissement de la production de protéines. La moyenne des essais récapitulés sur le tableau 2 fait état de baisses de production de protéines et de caséines de 25 et $27 \mathrm{~g} / \mathrm{j}$ respectivement. Cette tendance s'observe quelle que soit la nature de la ration et du supplément lipidique, ou le taux d'incorporation de ce dernier. Ainsi, elle est maintenue même lorsque le taux protéique du lot témoin a été accru par une infusion duodénale de lysine et méthionine (Chilliard et Doreau 1991). Il faut toutefois remarquer qu'il existe une tendance à la diminution du rapport caséines/protéines (- $0,8 \%$ sur la moyenne des essais du tableau 2 ).

La teneur en protéines solubles du lait ne varie pas en moyenne avec la supplémentation lipidique (tableau 2) les différences étant comprises entre - 0,7 g/kg (Chow et al 1990) et $+0,8 \mathrm{~g} / \mathrm{kg}$ (Horner et al 1986, Mohamed et al 1988) selon les essais. Les quantités de protéines solubles sécrétées sont d'ailleurs indépendantes de l'apport de lipides : celui-ci entraîne une variation journalière, positive ou négative, de $20 \mathrm{~g}$ au maximum.
La teneur en matières azotées non protéiques du lait ( $N$ non protéique $x 6,38$ ) ne varie pas en moyenne avec la supplémentation lipidique (entre $-0,2$ et $+0,2 \mathrm{~g} / \mathrm{kg}$ selon les essais). Ceci est logique, dans la mesure où cette fraction est en grande partie constituée d'urée, dont la concentration est voisine dans le lait et dans le sang. Cette dernière varie peu avec la supplémentation lipidique bien que la concentration d'ammoniac dans le rumen (qui est l'une des deux sources de l'urée sanguine) soit parfois réduite lors d'un apport important de matières grasses alimentaires (Doreau et al 1991). Il faut toutefois remarquer que, lorsque la teneur en azote non protéique dans le lait ne varie pas, sa part dans les matières azotées totales s'accroît de manière passive du fait de la diminution de la teneur en caséines.

\section{2 / Lipides de la ration et matières grasses du lait}

Les principales conséquences de l'incorporation de lipides dans les rations sur les matières grasses du lait seront rappelées dans ce texte. De plus amples détails pourront être trouvés dans les revues de Storry et al (1980) et Chilliard et al (1986).

\section{1 / Taux butyreux}

L'introduction de suppléments lipidiques dans les rations des vaches laitières entraîne soit un accroissement, soit pas de variation, soit une diminution du taux butyreux : ces tendances se manifestent respectivement dans 52,2 et $46 \%$ des observations d'après une synthèse de Morand-Fehr et al (1986) portant sur 66 essais menés essentiellement avec des matières grasses non protégées. L'effet est donc très variable et peut dépasser $10 \mathrm{~g} / \mathrm{l}$ d'accroissement ou de diminution.

L'augmentation du taux butyreux est d'autant plus forte que les rations sont pauvres en acides gras totaux (moins de $2 \%$ ). Au delà de $6 \%$ d'acides gras totaux (apportés sous forme non protégée) dans la ration, le taux butyreux tend à diminuer (Jarrige et Journet 1959, Chilliard et al 1986, Hermansen et Pedersen 1987).

Les accroissements de taux butyreux sont plus nombreux avec les matières grasses ne perturbant pas la digestion (pauvres en acides gras polyinsaturés) et, dans le cas des lipides riches en acides gras insaturés, avec les graines oléagineuses qu'avec les huiles végétales. Avec des matières grasses "protégées", l'effet sur le taux butyreux est toujours positif ou nul. Dans une synthèse de la bibliographie, Chilliard et al (1992) calculent un accroissement moyen de $5,2 \mathrm{~g} / \mathrm{l}$ avec des matières grasses encapsulées (52 essais) mais de $0,4 \mathrm{~g} / 1$ seulement avec des savons de calcium d'huile de palme (29 essais). 
Tableau 2. Influence d'une supplémentation lipidique sur les fractions azotées du lait de vache ${ }^{1}$

\begin{tabular}{|c|c|c|c|c|c|c|c|c|}
\hline \multirow{2}{*}{ Référence } & \multirow{2}{*}{$\begin{array}{l}\text { TP } \\
\text { Tém }\end{array}$} & \multicolumn{5}{|c|}{ Variations Lip-Tém } & \multicolumn{2}{|c|}{$\mathrm{C} / \mathrm{P}$} \\
\hline & & PL & $\mathrm{TP}$ & $\mathrm{TC}$ & PP & $\mathrm{PC}$ & Tém & Lip-Tém \\
\hline $1 \mathrm{a}$ & 30,2 & $-1,4$ & $-2,4$ & $-2,2$ & -95 & -83 & 83,1 & $-0,8$ \\
\hline $1 b$ & 31,2 & $-1,5$ & $-4,3$ & $-3,8$ & -141 & -122 & 82,7 & $-0,9$ \\
\hline 2 & 29,9 & $-0,7$ & $-3,3$ & $-3,9$ & -123 & -139 & 85,5 & $-3,9$ \\
\hline 3 & 29,5 & $+2,1$ & 0 & 0 & +62 & +50 & 80,7 & 0 \\
\hline 4 & 30,6 & $+1,0$ & $-1,0$ & $-0,8$ & +9 & 0 & 80,8 & 0 \\
\hline $5 a^{2}$ & 31,3 & $+0,4$ & $-1,1$ & $-1,4$ & -30 & -34 & 82,7 & $-1,5$ \\
\hline $5 b^{2}$ & 33,3 & $-2,1$ & $-1,3$ & $-2,1$ & -110 & -122 & 85,2 & $-3,0$ \\
\hline 6 & 31,2 & $+3,3$ & $-1,3$ & $-1,1$ & +73 & +55 & 80,1 & $-0,2$ \\
\hline $7 a$ & 34,5 & $+0,3$ & $-2,0$ & $-1,7$ & -43 & -37 & 80,0 & $-0,5$ \\
\hline $7 \mathrm{~b}$ & 30,3 & $-1,1$ & $+0,8$ & 0 & -13 & -30 & 81,8 & $-2,2$ \\
\hline 8 & 30,4 & $+2,4$ & $-1,5$ & $-1,1$ & +57 & +57 & 80,9 & $+0,4$ \\
\hline 9 & 28,8 & $+3,2$ & $-0,4$ & $-0,3$ & +72 & +56 & 79,9 & $+0,1$ \\
\hline $10 \mathrm{a}$ & 30,1 & $+1,1$ & $-2,2$ & $-1,9$ & -50 & -44 & 84,7 & 0 \\
\hline $10 \mathrm{~b}$ & 30,7 & $-1,3$ & $-1,2$ & $-1,2$ & -83 & -78 & 84,8 & $-0,5$ \\
\hline $11 \mathrm{a}$ & 28,0 & $+2,2$ & - 1,3 & $-0,9$ & +21 & +26 & 81,6 & $+0,2$ \\
\hline $11 \mathrm{~b}$ & 28,6 & $+0,8$ & $-1,2$ & $-0,5$ & -7 & -7 & 81,7 & $+0,6$ \\
\hline $12 \mathrm{a}$ & 30,9 & $+0,2$ & $-2,0$ & $-2,2$ & -75 & -74 & 83,5 & $-1,8$ \\
\hline $12 \mathrm{~b}$ & 33,3 & $-0,2$ & $-4,2$ & $-4,0$ & -115 & -107 & 83,8 & $-1,7$ \\
\hline $13 a$ & 30,9 & $+3,4$ & $-1,3$ & $-1,2$ & +64 & +50 & 83,2 & $-0,3$ \\
\hline $13 \mathrm{~b}$ & 32,0 & $+3,1$ & $-1,6$ & $-1,1$ & +38 & +38 & 82,7 & $+0,7$ \\
\hline
\end{tabular}

$\mathrm{PL}$ : production laitière $(\mathrm{kg} / \mathrm{j}) ; \mathrm{TP}:$ taux protéique $(\mathrm{g} / \mathrm{kg}) ; \mathrm{TC}:$ teneur en caséines $(\mathrm{g} / \mathrm{kg}) ; \mathrm{PP}:$ production de protéines $(\mathrm{g} / \mathrm{j})$

$\mathrm{PC}$ : production de caséines $(\mathrm{g} / \mathrm{j}) ; \mathrm{C} / \mathrm{P}$ : rapport caséines/protéines $(\%)$; Tém : régime témoin; Lip : régime enrichi en lipides.

1 - Storry et al $1974,10 \%$ d' huile de coprah ; a : ration normale, b : supplément de caséine

2 - Dunkley et al $1977,12 \%$ de suif protégé

3 - Ruegsegger et Schultz 1985, 2,5\% de lipides de graine de soja

4 - DePeters et al 1985, 3,8\% de lipides de graine de coton

5 - Horner et al 1986, 2,6\% de lipides de graine de coton ; a : ration normale, b : supplément de niacine

6 - DePeters et al $1987,7 \%$ de saindoux

7 - Mohamed et al 1988, a : $4 \%$ lipides de soja (huile ou graine), b : $4 \%$ lipides de coton (huile ou graine)

8 - DePeters et al 1989, 3,5\% de saindoux

9 - Kim et al 1989, $3 \%$ de lipides de graine de soja extrudée

10 - DePeters et Palmquist 1990, 2,9\% de savons de calcium d'huile de palme ; $a$ : ration normale, b : supplément de farine de poisson

11- Chow et al 1990, 4 \% de saindoux ; a : ration normale, b : supplément de lysine et méthionine protégées

12 - Chilliard et Doreau, 1991, 3,8\% d'huile de colza infusée dans le duodénum ; a : ration normale, b : infusion duodénale de lysine et méthionine

13 - Cant et al 1991, $4 \%$ de graisses animales ; infusion de caséine, a : dans le rumen, b: dans la caillette .

Certains chiffres, en particulier les productions journalières de protéines et caséines ont été calculés à partir de données parfois peu détaillées fournies dans les publications.

Données incomplètes. Hypothèse : $5 \%$ d'azote non protéique, indépendamment du régime.

Des effets similaires ont été obtenus chez la chèvre (Sauvant et al 1983). Chez la brebis, la supplémentation lipidique par des savons de calcium d'huile de palme a entraîné un accroissement du taux butyreux atteignant $18 \mathrm{~g} / \mathrm{l}$ (Caja et Guillou 1990).

\section{2 / Composition en acides gras du lait}

Les variations du taux butyreux résultent des variations de sécrétion des différents acides gras du lait relativement à la variation de production laitière. L'addition de lipides dans la ration se traduit presque toujours par une diminution de la sécrétion et de la teneur en acides gras à chaîne courte et moyenne, et une augmentation de la teneur en acides gras à longue chaîne dans le lait. Ceci est dû :
- à la fréquente augmentation relative d'acide propionique dans le mélange des acides gras volatils produits dans le rumen, aux dépens des acides acétique et/ou butyrique qui sont des précurseurs des matières grasses du lait (Bauchart et al 1985) ;

- à l'inhibition de la synthèse des acides gras courts et surtout moyens dans la mamelle par les acides gras longs (Chilliard $e t$ al 1981a), en particulier par les acides gras de structure trans (Banks et al 1984).

Parallèlement, les lipides alimentaires sont en partie transférés dans le lait, et accroissent donc la sécrétion d'acides gras longs. Le taux de transfert semble plus faible pour les acides gras à chaîne courte et moyenne (Storry et al 1974) certainement en raison des transformations qu'ils subissent dans le rumen ou dans 
le foie, et pour les acides gras à 20 et $22 \mathrm{C}$ (Pennington et Davis 1975).

Lorsque les lipides alimentaires sont protégés de l'hydrogénation dans le rumen par encapsulation par des protéines, ils sont absorbés sous forme insaturée. C'est sous cette forme qu'ils sont transférés dans le lait. Il est alors possible d'obtenir des laits de composition très particulière, atteignant $35 \%$ d'acide linoléique et, dans le cas de lait de chèvre, $25 \%$ d'acide linolénique, avec des suppléments respectifs de $8 \%$ environ d'huile de carthame et de $10 \%$ d'huile de lin (Cook et al 1972, Scott et Cook 1975). Toutefois, l'efficacité de la protection n'est pas toujours assurée, malgré l'appellation de "lipides protégés" (Doreau et al 1989).

Ces règles générales ne sont toutefois pas systématiquement vérifiées. Ainsi Schneider et al (1988) ont observé avec un supplément de savons de calcium d'huile de palme un accroissement de la sécrétion d'acides gras courts et moyens. Inversement, Doreau et al (1988 et non publié) ont signalé avec des suppléments d'huiles ou graines oléagineuses, l'absence totale d'augmentation de transfert d'acides gras longs dans le lait : les sécrétions d'acides gras à $18 \mathrm{C}$ avaient été respectivement de 350 et $313 \mathrm{~g} / \mathrm{j}$ avec les régimes témoin et enrichi en huile de soja. Ces cas particuliers n'ont pour l'instant pas d'explication.

\section{3 / Aptitude du lait à la transformation et conséquences pour le consommateur}

Les tendances à la diminution des teneurs en protéines et plus particulièrement en caséines du lait observées lors de supplémentation en lipides de la ration ont des conséquences négatives sur l'aptitude fromagère des laits (Remeuf et Hurtaud 1991). Par ailleurs, l'accroissement de la teneur en acides gras polyinsaturés dans le lait entraîne un accroissement de la durée de barattage lors de la fabrication des beurres, en particulier lorsque la température s'élève (Edmonson et al 1974). Les problèmes principaux spécifiquement liés à la distribution de quantités importantes de lipides dans la ration concernent la qualité organoleptique des produits (voir plus loin).

En revanche, la supplémentation en lipides des rations peut avoir des conséquences positives. Ainsi, une "tartinabilité" supérieure du beurre sortant du réfrigérateur est observée avec les laits riches en acides gras insaturés. Cette propriété, appréciée par le consommateur, s'ajoute à l'amélioration de la valeur diététique des produits, liée à la diminution de l'apport de graisses saturées et à l'accroissement éventuel des acides gras polyinsaturés. Il faut toutefois remarquer qu'à l'exception des huiles végétales encapsulées, qui permet- tent parfois d'augmenter les teneurs en acides gras polyinsaturés, on n'observe la plupart du temps qu'une augmentation des isomères monoinsaturés à 18 atomes de carbone, incluant des acides gras de structure trans susceptibles de poser certains problèmes diététiques (Emken 1984).

\section{1 / Altérations de la qualité organoleptique du lait liées à l'oxydation}

L'addition de lipides dans la ration a souvent été considérée comme préjudiciable aux qualités organoleptiques des produits laitiers, lorsque la composition des matières grasses du lait était modifiée. L'accroissement de la teneur en acide linoléique entraîne par ailleurs une tendance à l'oxydation et des altérations du goût.

Quelques études très complètes ont permis de situer l'importance des différents problèmes organoleptiques. Wong et al (1973), Edmondson et al (1974), Badings et al (1976) et Goering et al (1976), ayant obtenu des laits riches en acide linoléique (jusqu'à $30 \%$ des acides gras totaux) et parfois en acide linolénique, ont observé :

- une flaveur plus douceâtre des beurres et des fromages ; la qualité des fromages (type Gouda ou Cheddar) a toutefois été jugée "acceptable" jusqu'à $15 \%$ d'acide linoléique, malgré une consistance molle et farineuse, un goût parfois qualifié de "huileux", de "vieux" ou de "renfermé";

- une tendance à l'oxydation du beurre se traduisant par une dégradation du goût avec la durée de conservation ; celle-ci semble pouvoir être évitée par l'addition d'antioxydant dans le lait, mais pas dans l'alimentation des vaches. L'oxydation provoque par ailleurs une décoloration des fromages.

Un goût de "vieux" a également été observé avec des laits riches en acide oléique, sans accroissement du goût "oxydé" (Middaugh et al 1988). Il ne semble en revanche pas y avoir de problèmes d'oxydation de lait avec des suppléments en lipides non protégés (Lundin et Palmquist 1983, Schingoethe et al 1983, Finn et al 1985), puisque ceux-ci n'accroissent pas la teneur en acides gras polyinsaturés du lait. Seuls Perry et Macleod (1968) ont observé une légère détérioration de la flaveur du lait de vaches ayant reçu des graines de soja, bien que la composition ne soit que faiblement modifiée.

La consommation par les vaches de matières grasses oxydées n'a apparemment pas de conséquences négatives sur la composition et la flaveur du lait (Cadden et al 1984).

\section{2 / Lipolyse du lait et rancissement}

Parmi les détériorations de la qualité organoleptique des produits laitiers figure le rancissement, symptôme de lipolyse. La lipolyse, constituée par l'accroissement de la quantité 
d'acides gras libres présents dans le lait, peut être spontanée, c'est à dire liée aux composants du lait sortant de la mamelle, ou induite par des chocs thermiques ou mécaniques postérieurs à la récolte du lait. Les mécanismes et les cause de variation ont été décrits en détail par Chilliard et Lamberet (1984).

Des goûts de rance dans le lait apparaissent lorsque les vaches reçoivent de l'huile de coprah protégée (le lait est alors plus riche en acides gras courts et moyens) alors qu'ils ne sont pas observés avec des huiles de soja, de colza ou de carthame protégées (enrichissant le lait en acide linoléique) (Astrup et al 1976, $1980 \mathrm{a}$ et $1980 \mathrm{~b})$.

L'effet des lipides de la ration sur la lipolyse du lait (c'est-à-dire sa teneur en acides gras libres) a été analysé dans la revue de Chilliard (1982), qui confirme les données obtenues sur le rancissement du lait, à savoir une augmentation de la lipolyse lorsque la ration est enrichie en acide palmitique, mais pas lorsqu'elle l'est en acide stéarique (Astrup et al $1980 \mathrm{~b}$ ), et une réduction avec une supplémentation en huiles protégées (colza, carthame ou tournesol), enrichissant le lait en acide linoléique (Astrup et al 1979, Chilliard et al 1981b, Urquhart et al 1984). En revanche, un supplément d'huile de colza non protégée accroît la lipolyse (Chazal et Chilliard 1985). Les différentes hypothèses proposées pour expliquer ces résultats (modification de la structure des triglycérides, de la sécrétion de lipoprotéine lipase ou altération du passage d'activateurs sanguins dans le lait) n'ont pas été confirmées expérimentalement, sauf chez la chèvre, chez laquelle on observe une diminution de la lipase du lait (Chilliard et al 1981b).

\section{Conclusion}

L'intérêt de la supplémentation des régimes des vaches laitières en matières grasses n'est pas le même selon que l'objectif est l'accroissement de la production de lait ou l'amélioration de sa qualité. Il est certain que l'effet négatif des matières grasses alimentaires sur le taux protéique constitue un frein à leur emploi. Toutefois, les conditions optimales de leur utilisation (en fonction du stade de lactation notamment) ne sont pas encore parfaitement définies. Il est probable que l'utilisation des matières grasses dans l'alimentation des vaches laitières dépendra de l'équilibre entre différents facteurs économiques : coût relatif des matières grasses et des autres sources énergétiques, influence du taux protéique du lait sur son paiement à la production.

Ce texte a été présenté lors du colloque "Qualité des laits à la production et aptitude fromagère" organisé par l'INRA et l'ENSAR à Rennes, en janvier 1991.

\section{Références bibliographiques}

ASTRUP H.N., VIK-MO L., EKERN A., BAKKE F., 1976. Feeding protected and unprotected oils to dairy cows. J. Dairy Sci., 59, 426-430.

ASTRUP H.N., VIK-MO L., LINDSTAD P., EKERN A. 1979. Casein protected oil supplement fed at a low level to milk cows. Milchwissenschaft, 34, 290-291.

ASTRUP H.N., BAEVRE L., VIK-MO L., EKERN A., 1980a. Effect on milk lipolysis of restricted feeding with and without supplementation with protected rapeseed oil. J. Dairy Res., 47, 287-294.

ASTRUP H.N., VIK-MO L., SKROVSETH O, EKERN A., $1980 \mathrm{~b}$. Milk lipolysis when feeding saturated fatty acids to the cow. Milchwissenschaft, 35, 1-6.

BADINGS H.T., TAMMINGA S., SCHAAP J.E., 1976. Production of milk with a high content of polyunsatura ted fatty acids. II. Fatty acid composition of milk in relation to the quality of pasteurized milk, butter and cheese. Neth. Milk Dairy J., 30, 118-131.

BANKS W., CLAPPERTON J.L., GIRDLER A.K., STEELE W. 1984. Effect of inclusion of different form of dietary fatty acid on the yield and composition of cow's milk. J. Dairy Res., 51, 387-395.

BAUCHART D., DOREAU M., LEGAY-CARMIER F., 1985. Utilisation digestive des lipides et conséquences de leur introduction sur la digestion du ruminant. Bull. Tech. CRZV Theix, INRA, 61, 65-77.

BINES J.A., BRUMBY P.E., STORRY J.E., FULFORD R.J., BRAITHWAITE G.D., 1978. The effect of protected lipids on nutrient intakes, blood and rumen metabolites and milk secretion in dairy cows during early lactation. J. Agric. Sci. (Camb.), 91, 135-150.

CADDEN A.M., URQUHART A., JELEN P., 1984 Storage stability of canola-based protected lipid feed supplement and its effect on characteristics of milk and butter. J. Dairy Sci., 67, 1414-1420.

CAJA G., GUILLOU D., 1990. Alimentation des brebis laitières. Rôle des matières grasses protégées $(8 p$. Forum Volac, Paris

CANALE C.J., MULLER L.D., McCAHON H.A., WHIT SEL T.J., VARGA G.A., LORMORE M.J., 1990. Dietary fat and ruminally protected amino acids for high producing dairy cows. J. Dairy Sci., 73, 135-141.

CANT J.P., DEPETERS E.J., BALDWIN R.L., 1991 Effect of dietary fat and postruminal casein administration on milk composition of lactating dairy cows. J. Dairy Sci., 74, 211-219.

CASPER D.P., SCHINGOETHE D.J., 1989. Model to describe and alleviate milk protein depression in early lactation dairy cows fed a high fat diet. J. Dairy Sci., 72, 3327-3335

CASPER D.P SCHINGOETHE D J, EISENBEISZ W.A., 1990. Response of early lactation cows to diets that vary in ruminal degradability of carbohydrates and amount of fat. J. Dairy Sci., 73, 425-444

CHAZAL M.P., CHILLIARD Y., 1985. La lipolyse du lait ne résulte pas seulement du matériel de traite. Prod. Lait. Mod., 142, 52-53.

CHILLIARD Y., 1982. Variations physiologiques des activités lipasiques et de la lipolyse spontanée dans les laits de vache, de chèvre et de femme : revue bibliographique. Lait, 62, 1-31 et 126-154.

CHILLIARD Y., DOREAU M., 1991. Influence of a duodenal infusion of rapeseed oil and amino acids on cow milk yield and composition. Proc. 42nd Ann. Meet. Europ. Ass. Anim. Prod., Berlin, vol. 2, 26-27.

CHILLIARD Y., LAMBERET G., 1984. La lipolyse dans le lait: les différents types, mécanismes, facteurs de variation, signification pratique. Lait, $64,544-578$. 
CHILLIARD Y., SAUVANT D., MORAND-FEHR P., 1981a. Synthèse des matières grasses du lait chez les ruminants. Incidence de la conduite alimentaire et de l'utilisation des réserves corporelles $(45 \mathrm{p}$.). In "Physiologie de la sécrétion et de l'éjection du lait", ENSA Rennes.

CHILLIARD Y., SELSELET-ATTOU G., BAS P., MORAND-FEHR P., 1981b. Facteurs influençant la lipolyse du lait de chèvre. C.R. Gèmes J. Recherche ovine et caprine, Toulouse, 332-350, Ed. ITOVIC-SPEOC.

CHILLIARD Y., MORAND-FEHR P., SAUVANT D., BAS P., 1986. Utilisation métabolique des lipides par le ruminant en lactation. Bull. Tech. CRZV Theix, INRA, 63, 8191

CHILliaRD Y., DOREAU M., GAGLiOSTRO G., ELMEDDAH Y., 1992. Effets de la supplémentation en lipides protégés (lipides encapsulés et savons de calcium) sur les performances et le métabolisme des vaches laitières. INRA Prod. Anim., 5 , sous presse.

CHOW J.M., DEPETERS E.J., BALDWIN R.L., 1990. Effect of rumen-protected methionine and lysine on casein in milk when diets high in fat or concentrate are fed. J. Dairy Sci., 73, 1051-1061.

COOK L.J., SCOTT T.W., PAN Y.S., 1972. Formaldehyde-treated casein-safflower oil supplement for dairy cows. II. Effect on the fatty-acid composition of plasma and milk lipids. J. Dairy Res., 39, 211-218.

DEPETERS E.J., PALMQUIST D.L., 1990. Effect of fish meal and calcium salts of long chain fatty acids on the nitrogen content of milk. J. Dairy Sci., 73, suppl. 1, 242 (abstr.).

DEPETERS E.J., TAYLOR S.J., FRANKE A.A., AGUIRRE A., 1985. Effects of feeding whole cottonseed on composition of milk. J. Dairy Sci., 68, 897-902.

DEPETERS E.J., TAYLOR S.J., FINLEY C.M., FAMULA T.R., 1987. Dietary fat and nitrogen composition of milk from lactating cows. J. Dairy Sci., 70, 1192-1201.

DEPETERS E.J., TAYLOR S.J., BALDWIN R.L., 1989. Effect of dietary fat in isocaloric rations on the nitrogen content of milk from Holstein cows. J. Dairy Sci., 72, 2949-2957.

DOREAU M., CHILLIARD Y., BAUCHART D., MORAND-FEHR P., 1987. Besoins en lipides des ruminants. Bull. Tech. CRZV Theix, INRA, 70, 91-97.

DOREAU M., CHILLIARD Y., SAUVANT D., 1988. Effets d'une supplémentation en matières grasses sur l'ingestion et la production des vaches laitières. Symp. Franco-polonais "Systèmes d'alimentation azotée et utilisation des nutriments chez les ruminants", Varsovie, $6 \mathrm{p}$.

DOREAU M., FERLAY A., ELMEDDAH Y., BAUCHART D., 1989. La "protection" des matières grasses utilisées dans l'alimentation des ruminants : conséquences sur la digestion. Rev. Franç. Corps Gras, 36, 271-278.

DOREAU M., BEN SALEM H., KRZEMINSKI R., LEGAY F., BAUCHAR'T D., 1991. Ruminal nitrogen metabolism of cows fed diets supplemented or not with lipids. Proc. 6th Intern. Symp. Protein Metabolism and Nutrition, Herning, Danemark, vol. 2, 64-66.

DRIVER L.S., GRUMMER R.R., SCHULTZ L.H., 1990. Effects of feeding heat-treated soybeans and niacin to high producing cows in early lactation. J. Dairy Sci., 73, 463-469.

DUNKLEY W.L., SMITH N.E., FRANKE A.A., 1977. Effect of feeding protected tallow on composition of milk and milk fat. J. Dairy Sci., 60, 1863-1869.

EDMONDSON L.F., YONCOSKIE R.A., RAINEY N.H., DOUGLAS Jr F.W., BITMAN J., 1974. Feeding encapsulated oils to increase the polyunsaturation in milk and meat fat. J. Am. Oil Chem. Soc., 51, 72-76.

EMKEN E.A., 1984. Nutrition and biochemistry of trans and positional fatty acid isomers in hydrogenated oils. Ann. Rev. Nutr., 4, 339-376.

FINN A.M., CLARK A.K., DRACKLEY J.K., SCHINGOETHE D.J., SAHLU T., 1985. Whole rolled sunflower seeds with or without additional limestone in lactating dairy cattle rations. J. Dairy Sci., 68, 903-913.
GAGLIOSTRO G., CHILLIARD Y., 1991. Duodenal rapeseed oil infusion in early and mid-lactation cows. 2 Voluntary intake, milk production and composition. J. Dairy Sci., 74, 499-509.

GAGLIOSTRO G., CHILLIARD Y., DAVICCO M.J., 1991. Duodenal rapeseed oil infusion in early and mid-lactation cows. 3. Plasma hormones and mammary apparent uptake of metabolites. J. Dairy Sci., 74, 1893-1903.

GOERING H.K., GORDON C.H., WRENN T.R., BITMAN J., KING R.L., DOUGLAS Jr F.W., 1976. Effect of feeding protected safflower oil on yield, composition, flavor, and oxidative stability of milk. J. Dairy Sci., 59, 416-425. HERMANSEN J.E., PEDERSEN J., 1987. Milk composition in relation to breeding and feeding. In "Research in cattle production. Danish status and perspectives" Landhusholdningsselskabets Forlag., Copenhague, pp. 140-154.

HOFFMAN P.C., GRUMMER R.R., SHAVER R.D., BRODERICK G.A., DRENDEL T.R., 1991. Feeding supplemental fat and undegraded intake protein to early lactation dairy cows. J. Dairy Sci., 74, 3468-3474.

HORNER J.L., COPPOCK C.E., SCHELLING G.T. LABORE J.M., NAVE D.H., 1986. Influence of niacin and whole cottonseed on intake, milk yield and composition, and systemic responses of dairy cows. J. Dairy Sci., 69, 3087-3093

JARRIGE R., JOURNET M., 1959. Influence des facteurs alimentaires et climatiques sur la teneur en matières grasses du lait. Ann. Nutr. Alim., 13, A233-A277.

KIM Y.K., SCHINGOETHE D.J., CASPER D.P., LUDENS F.C., 1989. Lactational response of dairy cows to diets containing increased amounts of crude protein with increased amounts of energy from added fat. J. Dairy Sci., 72, suppl. 1, 548 (abstr.).

LOUGH D.S., MULLER L.D., KENSINGER R.S., SWEENEY T.F., GRIEL JR L.C., 1988. Effect of added dietary fat and bovine somatotropin on the performance and metabolism of lactating dairy cows. J. Dairy Sci., 71, 1161-1169

LUNDIN P.K., PALMQUIST D.L., 1983. Vitamin E supplementation of high fat diets for dairy cows. J. Dairy Sci., 66, 1909-1916.

MACLEOD G.K., YU Y., SCHAEFFER L.R., 1977. Feeding value of protected animal tallow for high yielding dairy cows. J. Dairy Sci., 60, 726-738.

MIDDAUGH R.P., BAER R.J., CASPER D.P., SCHIN GOETHE D.J., SEAS S.W., 1988. Characteristics of milk and butter from cows fed sunflower seeds. J. Dairy Sci., 71, 3179-3187

MOHAMED O.E., SATTER L.D., GRUMMER R.R. EHLE F.R., 1988. Influence of dietary cottonseed and soybean on milk production and composition. J. Dairy Sci., 71, 2677-2688.

MORAND-FEHR P., CHILLIARD Y., BAS P., 1986. Répercussions de l'apport de matières grasses dans la ration sur la production et la composition du lait de ruminant. Bull. Tech. CRZV Theix, INRA, 64, 59-72.

PALMQUIST D.L., MOSER E.A., 1981. Dietary fat effects on blood insulin, glucose utilization and milk protein content of lactating cows. J. Dairy Sci., 64, 16641670.

PENNINGTON J.A., DAVIS C.L., 1975. Effects of intraruminal and intra-abomasal additions of cod-liver oil on milk fat production in the cow. J. Dairy Sci.., 58, 49-55.

PERRY G.F., MACLEOD G.K., 1968. Effects of feeding raw soybeans on rumen metabolism and milk composition of dairy cows. J. Dairy Sci., 51, 1233-1238.

REMEUF F., HURTAUD C, 1991. Liaisons entre les caractéristiques physico-chimiques des laits et leurs aptitudes fromagères. In: Qualité des laits à la production et aptitude fromagère, Ed. M. Journet, A. Hoden, G. Brulé, INRA-ENSA Rennes, 15pp.

RUEGSEGGER G.J., SCHULTZ L.H., 1985. Response of high producing dairy cows in early lactation to the feeding of heat-treated whole soybeans. J. Dairy Sci., 68, $3272-3279$. 
SAUVANT D., MORAND-FEHR P., BAS P., 1983. L'intérêt des lipides dans les aliments concentrés: observations chez la chèvre. In CAAA "Quels aliments concentrés pour les vaches fortes productrices de lait?", K1-K17, Ed. ADEPRINA, Paris.

SCHINGOETHE D.J., STEWART G.D., LUDENS F.C., 1983. Dried whey-fat blend product as a feed for lactating dairy cows. J. Dairy Sci., 66, 2515-2520.

SCHNEIDER P. SKLAN D., CHALUPA W., KRONFELD

D.S., 1988. Feeding calcium salts of fatty acids to lactating cows. J. Dairy Sci., 71, 2143-2150.

SCOTT T.W., COOK L.J., 1975. Effect of dietary fat on lipid metabolism in ruminants. In "Digestion and metabolism in the ruminant" (I.W. McDonald et A.C.I. Warner, éd.), pp. 510-532, Univ. New England, Armidale, Australie.

STORRY J.E., BRUMBY P.E., HALL A.J., JOHNSON

V.W., 1974. Response of the lactating cow to different methods of incorporating casein and coconut oil in the diet. J. Dairy Sci., 57, 61-67.

STORRY J.E., BRUMBY P.E., DUNKLEY W.L., 1980. Influence of nutritional factors on the yield and content of milk fat: protected non-polyunsaturated fat in the diet. Int. Dairy Fed. Bull., 125, 105-125.

TAMMINGA S., DOREAU M., 1990. Lipids and rumen digestion. In "Rumen microbial metabolism and ruminant digestion", INRA Clermont-Ferrand - Theix.

URQUHART A., CADDEN A.M., JELEN P., 1984. Quality of milk and butter related to canola-based protected lipid feed supplement. Milchwissenschaft, 39, 1-6.

WONG N.P., WALTER H.E., VESTAL J.H., LACROIX D.E., ALFORD J.A., 1973. Cheddar cheese with increased polyunsaturated fatty acids. J. Dairy Sci., 56, 12711275

\section{Summary}

Fat supplementation of dairy rations and its consequences on milk quality in dairy cows.

Fat supplementation of dairy rations is often practised. This decreases milk protein content, especially the casein fraction. The causes are up till now not well known. A part of this effect is related to the dilution of the proteins in a higher milk volume since total protein secretion is not greatly altered. The decrease in protein content is very slight in early lactation where the positive effect on milk yield is less marked than in mid-lactation.

Fat supplementation has a variable effect on butterfat. This effect depends on the balance between the increase in dietary long-chain fatty acid uptake by the udder and the decrease in mammary synthesis of short-and medium-chain fatty acids. The content of milk in polyunsaturated fatty acids is in most cases not increased, due to ruminal hydrogenation of dietary fatty acids. Encapsulation of lipids by protected proteins is the only technique which prevents ruminal hydrogenation of polyunsaturated fatty acids.

Some kinds of lipid supplementation lead to a lowering of the organoleptic value of dairy pro. ducts, especially through oxidation and rancidity.

DOREAU M., CHILLIARD Y., 1992. Influence d'une supplémentation de la ration en lipides sur la qualité du lait chez la vache. INRA Prod. Anim., 5 (2), 103 - 111. 